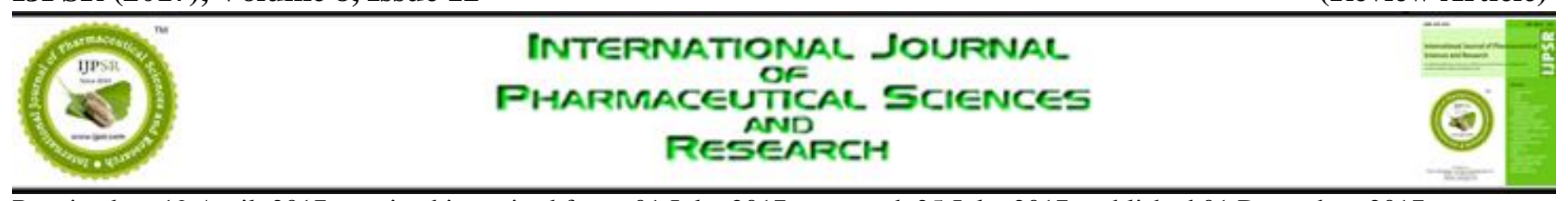

Received on 19 April, 2017; received in revised form, 01 July, 2017; accepted, 25 July, 2017; published 01 December, 2017

\title{
A REVIEW ON NANOPARTICULATE MUCOADHESIVE SYSTEM
}

\author{
Gouri Shankar* and Y. K. Agrawal
}

Institute of Research and Development, Gujarat Forensic Sciences University, DFS Head Quarters, Sector 18-A, Near Police Bhavan, Gandhinagar - 382007, Gujarat, India.

Keywords:

Mucoadhesion, GIT, NPs, Peptides, Drug delivery

\section{Correspondence to Author:}

Gouri Shankar

Drugs Inspector,

Institute of Research and

Development, Gujarat Forensic

Sciences University, DFS Head

Quarters, Sector 18-A, Near Police

Bhavan, Gandhinagar - 382007,

Gujarat, India.

E-mail: gourishankarthakur@yahoo.com

\begin{abstract}
Peptide drugs are poorly absorbable and are also susceptible to enzymatic degradation in the gastrointestinal tract (GIT). To overcome the physiological and morphological barriers for the peptide / poorly absorbable drug delivery, mucopenetrative and mucoadhesive properties of drug carriers have to be considered. Mucoadhesion can prolong the residence time of drug carriers at the absorption sites, improved drug absorption and controlled release of drug from the device used. The particles with smaller size were likely to diffuse much faster into the mucus layer, provided that diffusion of the particles into the mucus layer should obey Stokes-Einstein equation. The adhesiveness of the nanoparticles (NPs) in suspension form helps to improve bioavailability. Chitosan (CS) based or CS-coated NPs represent another common group of mucoadhesive systems. Moreover, NPs made of such polymers were used to enhance the bioavailability, and also the mucoadhesion of proteins, peptides and DNA to the mucosal tissues. This review focuses on the mechanism of gastrointestinal mucoadhesion of NPs for peptide/poorly absorbable drugs.
\end{abstract}

\section{INTRODUCTION: Size reduction helps in} improving solubility and bioavailability, reducing toxicity, enhancing release and providing better formulation opportunities for drugs. Drugs in the nanometer size range enhance performance in a variety of dosage forms. The word 'nano' was derived from a Latin word, which means dwarf. Nano size refers to one thousand millionth of a particular units (i.e. $1 \mathrm{~nm}=10^{-9} \mathrm{~m}$ ) ${ }^{1}$. The development of a dosage form to improve the oral absorption of peptides and proteins or any poorly absorbed drug, whose bioavailability was very low because of instability in the gastrointestinal (GI) tract and low permeability through the intestinal epithelium, was one of the greatest challenges in the pharmaceutical field ${ }^{2}$.

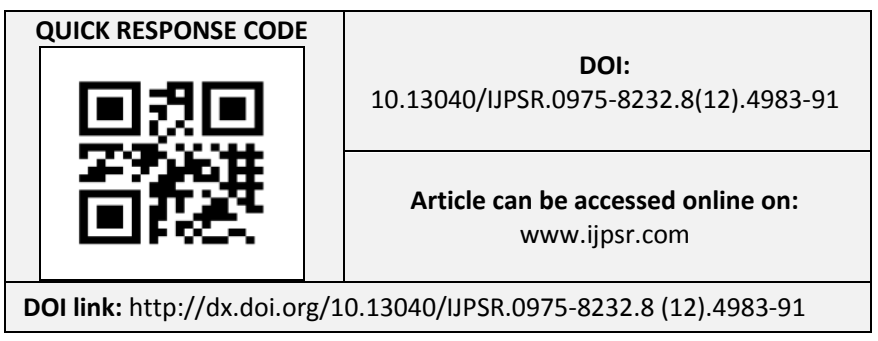

The bio-adhesion of the oral dosage forms of poorly absorbable drugs has received much attention in transdermal and buccal systems. Bioadhesion to mucous membranes in the gastrointestinal tract can be described in term of mucoadhesion as it can prolong the residence time of drug carriers at the absorption sites.

Longer and group were the first to show that a delayed gastrointestinal transit induced by mucoadhesive polymers could lead to the increased oral bioavailability of a drug. Peptide drugs were intrinsically poorly absorbable owing to their high molecular weight and hydrophilicity; they are also susceptible to enzymatic degradation in the gastrointestinal tract ${ }^{3}$. The drug carriers are expected to remain in the gastrointestinal tract while protecting the entrapped peptide drugs from enzymatic degradation until they are released and absorbed. The concept of this system could be also applied to other peptide delivery systems, such as nasal and pulmonary administration systems via the mucous membranes ${ }^{4}$. 
1.1. Mucoadhesion of the Nanoparticles: Nanoparticles (NPs) orally administered in the form of a suspension diffuse into the liquid media and rapidly encounter the mucosal surface. The particles are immobilized at the intestinal surface by an adhesion mechanism referred to as "bioadhesion". The concentrated suspension acts as a reservoir of particles and an adsorption process takes place very rapidly. The direct contact of the particles with the intestinal cells through a bioadhesive phase is the first step before particle absorption. The adhesiveness of the nanosuspensions not only helps to improve bioavailability but also improves targeting of the parasites persisting in the GIT. Bupravaquone nanosuspensions have been reported to demonstrate an advantage in TRC-alpha-deficient mice infected with Cryptosporidium parvum oocytes ${ }^{5,6,27,46}$.

\subsection{Mucoadhesion of NPs after Oral} Administration: Oral administration of polymeric particle suspensions (NPs or micron-range microspheres made from non-swellable polymers) leads to mucoadhesion of a significant fraction of the particles. Different phenomena occur after oral administration (Fig. 1). Firstly, a suspension of particles is administered and immediately enters in contact with a portion of the oral mucosa (step I). From this moment, the concentrated suspension acts as a reservoir of particles and very rapidly, an adsorption process takes place, leading to the adsorption of a fraction of the available particles (step II). Adsorption occurs with the mucus layer and is an irreversible process. The luminal particle suspension transits through the intestine, sweeping progressively the whole mucosa. The simultaneous adsorption process results in a progressive covering of the intestinal mucosa by adherent particles (step III). Finally, detachment of the particles from the mucosa begins to occur in the proximal region and is progressively extended to the distal region (step IV) as shown in Fig. $\mathbf{1}^{5}$.

The inherent short comings of conventional drug delivery and the potential of NPs as drug delivery systems have offered a tremendous scope for researchers in this field and is fast moving from concept to reality. NPs may be used for oral administration of gut-labile drugs or those with low aqueous solubility ${ }^{7}$. These colloidal carriers have the ability to cross the mucosal barrier as such. In addition to the potential for enhancing drug bioavailability via particle uptake mechanisms, nanoparticulate oral delivery systems also have slower transit times than larger dosage forms increasing the local concentration gradient across absorptive cells, thereby enhancing local and systemic delivery of both free and bound drugs across the gut. These colloidal carriers are expected to develop adhesive interactions within the mucosa and remain in the gastrointestinal tract ${ }^{8}$.

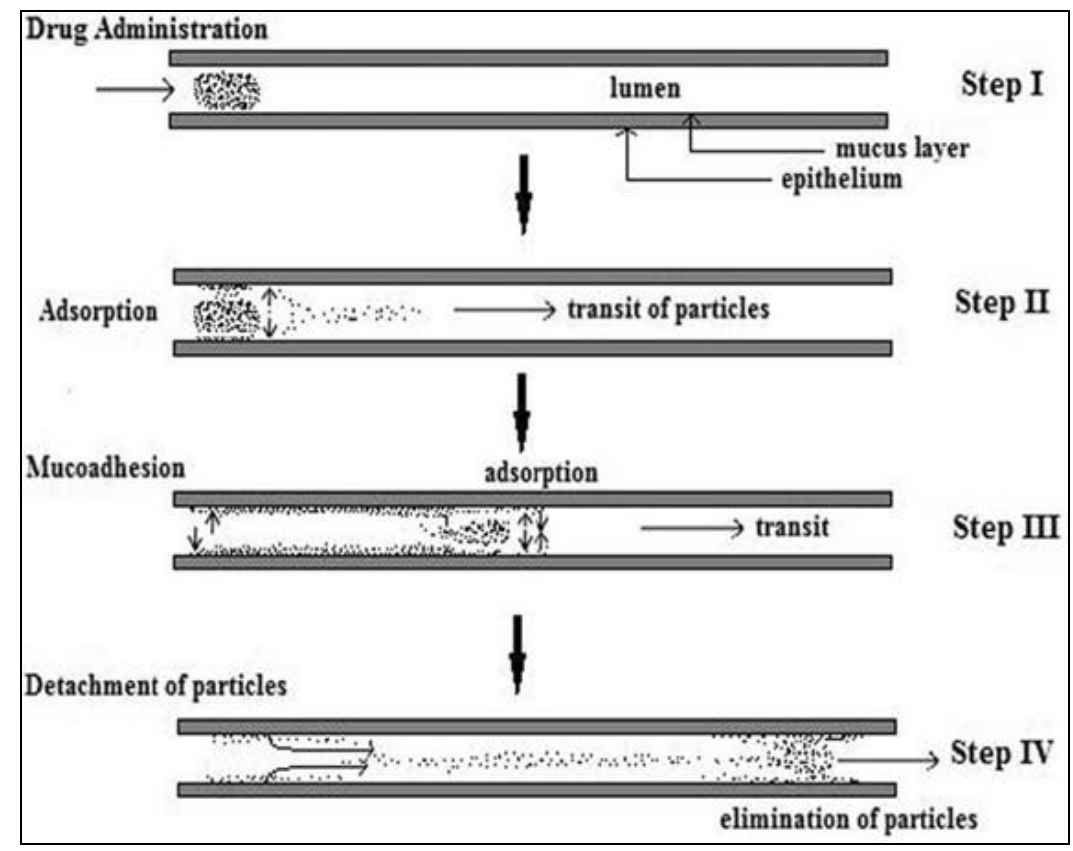

FIG. 1: MUCOADHESIVE BEHAVIOR OF COLLOIDAL PARTICULATE SYSTEMS FOLLOWING ORAL ADMINISTRATION 
1.3. Gastrointestinal Tract (GIT) Physiology with Relevance to Particulate Uptake: The GIT serves to carry out the digestion of food and the absorption of water, nutrients, and electrolytes, and provides a selective barrier between the environment and the systemic circulation. It provides a variety of physiological and morphological barriers, such as proteolytic enzymes, in the gut lumen and at the brush border membrane: the mucous layer, the bacterial gut flora, and the epithelial cell lining itself. Although the GIT is designed (evolved) to prevent uptake of particulate matter (e.g. potentially toxic materials and pathogens) from the environment, it is not a completely prohibitive barrier ${ }^{9-11}$. The chemical composition of mucus provides an opportunity for both acidic and basic compounds to interact with it, thereby increasing the residence time of both drugs and NPs in close proximity to the absorptive surfaces.

The primary function of the GIT is to selectively take up interest substances from the ingested bulk. To prevent harmful material from getting in, various protective mechanisms like $\mathrm{pH}$ variation, degrading enzymes, mucus, and non-pathogenic microflora exist. Also, the immunological load ingested makes the mucosa an ideal site for the identification and resistance of antigenic challenges. The local immune system is composed of GALT (gut associated lymphoid tissues), composed of lymphoid tissues, called PPs in the small intestine, which are characterized by a monolayer of specialized epithelium containing $\mathrm{M}$ cells and absorptive enterocytes, i.e. follicle associated epithelium (FAE) ${ }^{12}$.

1.4. Channels of Uptake: To deliver the drug content into the blood, lymph, or target organs, NPs have to cross the gastrointestinal barrier either by passive diffusion via transcellular or paracellular pathways or by active processes mediated by membrane bound carriers or membrane-derived vesicles ${ }^{13,14}$. Another possible mechanism for the transport of NPs across intestinal cells is paracellular uptake via aqueous channels. In humans, the equivalent pore diameter has been estimated to be between 4 and $8 \dot{A}$ and about 10-15 $\dot{A}$ in rat and rabbit. The mucosal epithelium in the small intestine consists of polarized cells, connected by tight intercellular junctions, which account for $<1 \%$ of the surface area of the intestine ${ }^{15}$. Fig. 2 represents schematic representation of the mechanism of uptake of NPs on oral administration. The direct uptake of NPs through the lymph into the systemic circulation by passing the liver reduces the first pass metabolism, thus improving bioavailability ${ }^{12}$.

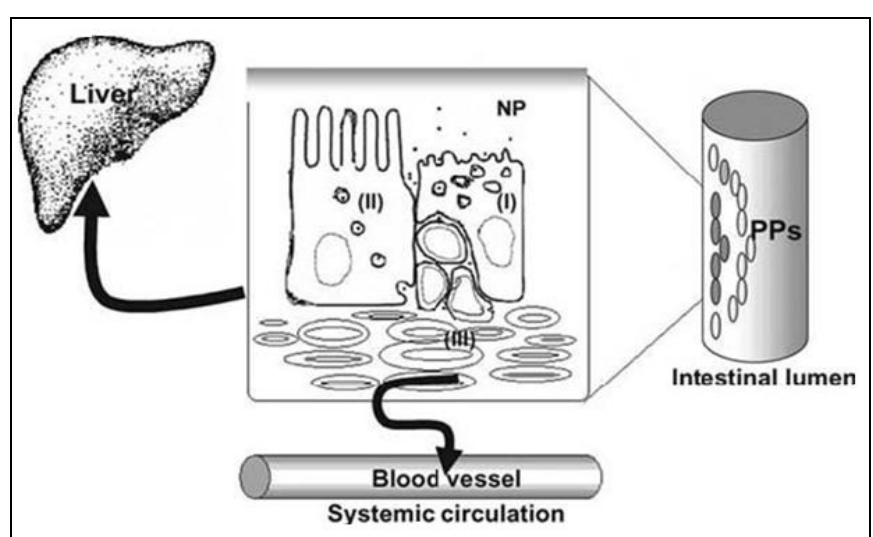

FIG. 2: MECHANISM OF UPTAKE OF ORALLY ADMINISTERED NPS

2. Bioadhesion: The particles come into contact with the mucosal surface; they have to come in direct contact with mucus and may develop interactions with it. When microparticles or NPs are orally administered in the form of a suspension, they diffuse into the liquid medium and encounter the mucosal surface rapidly during the time course of their transit in the GIT, thus remaining away from the absorbing surfaces for most of the time. The particles can be immobilized at the intestinal surface by an adhesion mechanism, referred to as bioadhesion ${ }^{16}$. Bioadhesion can be achieved by either nonspecific or specific interactions with the mucosal surface.

Nonspecific interactions are driven by the physicochemical properties and surface of the particles. Specific interactions depend on the presence of a ligand attached to the particle used for the recognition and attachment to a specific site at the mucosal surface. The process involved in the formation of such bioadhesive bonds has been described in three steps: (i) wetting and swelling of the polymer to permit intimate contact with biological tissue, (ii) interpenetration of bioadhesive polymer chains and entanglement of polymer and mucin chains, and (iii) formation of weak chemical bonds ${ }^{17}$. In 1989, Pappo generated a monoclonal antibody that recognized $\mathrm{M}$ cells of rabbit's PPs ${ }^{18}$. 
This monoclonal antibody was adsorbed onto fluorescent polystyrene microspheres, and the particles localized specifically in the PP epithelium after administration. Antibody coated particles accumulated in thrice the amount in PPs as compared to uncoated ones. The concept of site specificity for mucoadhesion based on the affinity between sugars and lectins has also been intensively investigated ${ }^{12}$. Wheat germ agglutinin (WGA) from Triticum vulgare specifically binds to $\mathrm{N}$-acetyl-D glucosamine and sialic acid, both of which are constituents of mucus. In addition to binding to the surface of Caco- 2 cells and human enterocytes, WGA is also taken up into the cells by receptor mediated endocytosis involving the epidermal growth factor (EGF) receptor, which is present in a significant amount even on enterocytes. Poly(lactide-co-glycide) (PLGA) NPs conjugated with WGA as a carbohydrate binding ligand showed improved cytoassociation as compared to the unmodified ones $19,27,46$.

Mucus is continuously secreted, then shed and discarded or digested and recycled. Its lifetime is short, often measured in minutes to hours. The fastest turnover is typically observed at surfaces with the thinnest mucus layer, perhaps as an evolutionary response to improve the protective features of the mucosa. In the nasal tract, the ciliary motion transports mucus from the anterior towards the posterior region of the nasal cavity, moving entrapped particles and potentially toxic materials toward the nasopharynx. The mucus flow rate is about $5 \mathrm{~mm}$ per min, and the mucus layer is renewed approximately every $20 \min ^{20-22}$.

Likewise, the luminal gel layer of respiratory tract mucus is replaced every 10 to $20 \mathrm{~min}$, leading to efficient clearance of inhaled particulates ${ }^{23}$. In contrast, the sol phase of respiratory mucus is thought to be cleared much less rapidly than the more solid-like luminal gel layer ${ }^{24-27}$. Continuous secretion and clearance by peristaltic forces leads to quick turnover times, on the order of $4-6 \mathrm{~h}$, in the rat GIT 7, 28, 29 and likely similar values in humans. In the vagina, mucus is cleared (expelled through the introitus) by intra-abdominal pressure as well as abdominal motions, which squeeze the walls of the vagina together ${ }^{30,}{ }^{31}$. Table $\mathbf{1}$ represents Thickness of various types of normal human mucus.
TABLE 1: THICKNESS OF VARIOUS TYPES OF NORMAL HUMAN MUCUS

\begin{tabular}{ccc}
\hline $\begin{array}{c}\text { Type of } \\
\text { Mucus }\end{array}$ & $\begin{array}{c}\text { Average } \\
\text { Thickness }(\boldsymbol{\mu m})\end{array}$ & $\begin{array}{c}\text { Reported } \\
\text { Thicknesses }(\boldsymbol{\mu m})\end{array}$ \\
\hline Respiratory: & & \\
Airway 50-52 & 15 & $7 ; 30$ \\
Bronchial 53 & 55 & $55 \pm 5$ \\
Gastrointestinal: & & \\
Ileal 55-58 & 10 & 10 \\
Cecal 56 & 37 & $36.7 \pm 7.2$ \\
Colonic 54-56,61 & 100 & $39.1 \pm 9.9(\mathrm{~A})$, \\
& & $67.5 \pm 14.5(\mathrm{~T})$, and \\
& & $79 \pm 40 ; 100-150 ;$ \\
& & $107 \pm 48$ \\
Rectal 55-57 & 125 & $101.5 \pm 80.3 ;$ \\
& & $155 \pm 54$ \\
Ocular: & & $0.02-0.05$ \\
Mucus layer 58 & 0.035 & $3 ; 6-7 ; 34-45$ \\
Tear film 59 & $5 ; 40$ & \\
\hline
\end{tabular}

\subsection{Increased Oral Bioavailability after Drug} Delivery by NPs: The pharmacokinetics of several drugs after oral administration has been improved by means of NPs. The bioavailability of vincamine was about $25 \%$ when administered in an aqueous solution to rabbits. After oral administration of vincamine adsorbed on poly (hexyl cyanoacrylate) NPs, the bioavailability reached $40 \%$, probably due to a prolonged period of contact of the drug delivery system with the mucosa ${ }^{32}$. Nanocapsules of poly (isobutyl cyanoacrylate), administered in the jejunal lumen, increased the absorption of Lipiodol: the plasma level of iodine was maximal 45 min after the administration of Lipiodol emulsion (3 times basal value) and nanocapsules (3.5 times). Then iodemia decreased again but remained 2.5-fold higher than control values 105 min after the administration of nanocapsules. The observation was attributed to a prolonged time period of contact between the lipiodol drug and the microvilli of mucus membrane ${ }^{33}$.

2.2. Behaviours of NPs in the GIT: Orally administered nanoparticulate carriers should have an ability to protect the loaded peptides from the digestive enzymes, such as pepsin, in the stomach with acidic conditions while they do not release the loaded peptides. Followed by passing through the stomach, the nanocarriers reach the small intestine that is a major absorption site for peptide drugs. Here, two major biological barriers for the drug absorption exist, i.e. the brush border membrane and mucus layer. 
Mucus is a fully hydrated viscoelastic gel (water occupies almost $95 \%$ of the contents under nonpathological conditions) overlying epithelial cell surfaces as a continuous gel blanket ${ }^{34-36}$. Its neogenesis continuously takes place with relatively rapid turnover rate ${ }^{37}$. Modification of nanoparticle surface with mucoadhesive components may lead to further prolongation of the residence time at the absorption site. Drug release from the NPs at the epithelial cell lining followed by penetration of the NPs into the depths of the mucus layer can give rise to high drug concentration gradient across the intestinal membrane, possibly leading to the increased drug absorption by passive diffusion.

Alternative approach is to directly traverse the NPs themselves to the blood stream through the intestinal membrane. This approach makes it possible to protect peptide drugs from proteolytic degradation by digestive enzymes such as trypsin and chemotripsin existing in the intestinal mucus layer. As transport pathways through the intestinal membrane, three possible routes have been suggested: (1) uptake by the PPs, (2) gap between epithelial absorptive enterocytes (paracellular route), (3) uptake by absorptive enterocytes (transcellular route) ${ }^{38}$.

3. Natural Polymers and Derivatives: The use of colloidal carriers made of hydrophilic polysaccharides like chitosan (CS) is increasing as a promising alternative for improving the transport of drugs and macromolecules, such as peptides, proteins, oligonucleotides, and plasmids across biological membranes. CS [(1- $\beta$-4)-2-amino-2deoxy- $\beta$-D-glucan] is a deacetylated chitin that has gained considerable interest for oral drug delivery. CS has been shown to increase the paracellular permeability of (14C) mannitol (a marker for paracellular routes) across Caco-2 intestinal epithelia ${ }^{39}$. These findings attributed the property of transmucosal absorption enhancement. CS is soluble only in solutions at $\mathrm{pH}$ values below 6.5 , and only protonated CS (i.e. in its uncoiled configuration) can open the tight junctions, thereby facilitating the paracellular transport of hydrophilic compounds.

The problem of CS ineffectiveness at neutral $\mathrm{pH}$ values can be tackled by derivatization at the amine group that renders the polymer soluble and effective for the purpose ${ }^{40}$. However, $\mathrm{pH}$ above 6.5 is encountered only at the distal ends of the enteron and is expected to be of concern only when NPs are targeted to these portions of the GIT, such as the colon. As CS easily forms NPs and microparticles with high loading capacities for various antigens, it is a promising candidate for designing carrier systems for oral vaccine delivery. An important advantage of CS NPs and microparticles is that, often, the use of organic solvents, which may alter the immunogenicity of antigens, is avoided during preparation and loading ${ }^{41}$.

The potential of certain bioadhesive NPs have evaluated to increase the oral bioavailability of drugs degraded in GIT using 5-fluorouridine as a model drug. From the urine data, poly (methylvinylether-co-maleic anhydride) NPs and those coated with albumin showed higher bioavailability over the control oral solution ${ }^{42}$. A bupravaquone mucoadhesive nanosuspension was produced using an optimal stabiliser combination and incorporating the nanosuspension in a mucoadhesive gel. Preparation of this mucoadhesive nanosuspension gel in the 'classical way' requires a three step production process that means producing the nanosuspensions, producing the gel and finally mixing the two components.

Alternatively, a more straightforward process for the general production of mucoadhesive nanosuspension gels was developed. The drug powder was dispersed in the surfactant solution as described above, and then the polymer was added and partially hydrated by addition of triethanolamine (adjustment of $\mathrm{pH}$ to approximately 5). This leads to a slightly viscous system which could still be passed through the homogeniser at two cycles 150 bar, two cycles 500 bar (premilling process) and then for 15 cycles applying 1500 bar.

Then the remaining triethanolamine was added to adjust to a $\mathrm{pH}$ of 7 to form the highly viscous gel (addition of triethanolamine under stirring using an ultra turrax). Nanosuspension technology and the mucoadhesive principle were combined to develop a formulation for the poorly soluble drug bupravaquone, especially for the treatment of Cryptosporidium parvum infections $27,44-46$. 


\section{Important Parameters of Polymeric Nanoparticles:}

\subsection{Preparation Methods for Polymeric NPs:}

4.1.1. Emulsion Solvent Diffusion Method: Emulsion solvent diffusion (ESD) methods can be classified into two categories: the "in water" method and the "in oil" method. To prepare PLGA NPs by the ESD in water method, PLGA and drug are dissolved in a mixed solvent of acetone and ethanol (or methanol), both of which dissolve in aqueous solution. When this polymer solution is poured into aqueous poly (vinyl alcohol) (PVA) solution, a submicronized o/w emulsion is spontaneously formed due to immediate reduction of the interfacial tension with rapid diffusion of organic solvent into the aqueous phase (the Marangoni effect). Following solvent diffusion, the nanospheres are obtained by co-precipitation of polymer and drug following the reduction in solubility.

During preparation of the NPs, PVA prevents the aggregation and fusion of emulsion droplets by adsorbing on the surface of the droplets. Then, the resultant nanosphere suspension is centrifuged and resuspended in distilled water to remove the solvent and free drug in the upper aqueous phase. Finally, the nanospheres are powered by freezedrying. In the "water" method, the emulsion is spontaneously submicronized due to the rapid solvent diffusion at the surface of droplets. In the "oil" method, however, such rapid diffusion does not occur. Therefore, there is a need to add a surfactant (Span 80) to the polymer phase in the in oil method. Nanospheres with an average diameter of ca. $700 \mathrm{~nm}$ were obtained. The polydispersity index of this suspension ranges from 0.2 to $0.3^{44-49}$.

4.1.2. Phase Separation Method: The phase separation method was devised to modify the drug encapsulation properties. In this method, aqueous drug solution is poured into a dichloromethane / acetone solution containing PLGA which is being agitated using a high-shear homogenizer. After preparation of the w/o emulsion, MCT is added to the system while stirring to induce phase separation. The coacervated droplets of PLGA surround the drug aqueous droplets due to the high activation energy of the surface of the aqueous droplets. Following the phase separation process, PLGA is precipitated by removing the organic solvent under vacuum. The nanospheres prepared by the phase separation method have a bimodal particle size distribution with a weight mean diameter and polydispersity index of $800 \mathrm{~nm}$ and $0.3-0.5$, respectively, due to a degree of coalescence of coacervated droplets ${ }^{50}$.

\subsection{Surface Modification of Nanoparticulate Systems with Mucoadhesive Polymers: One of} the most promising strategies for designing mucoadhesive particulate systems is surface modification, or coating, of the drug carrier particles with mucoadhesive polymers. For coarse particles, a conventional coating method, such as fluidized bed coating, can be used. In reviewing the surface modification of colloidal particles, a number of investigations of the polymer coating of liposomes have been published ${ }^{51}$.

\subsection{Evaluation of Mucoadhesive Particulate} Systems: Various methods have been proposed to evaluate the mucoadhesive properties of tablets or mucoadhesive polymers themselves ${ }^{52}$. In particular, special methods may be required to evaluate the mucoadhesive properties of nanoparticulate systems. The mucoadhesive properties of particulate systems have been evaluated ${ }^{53}$. The particles were prepared by coating glass beads $(0.45-0.5 \mathrm{~mm})$ or crystals of acetyl salicylic acid $(>630 \mu \mathrm{m})$ with various hydrophilic polymers such as Polycarbophil (PC), Sodium carboxymethyl cellulose (NaCMC), Hydroxypropylmethyl cellulose (HPMC), Methyl cellulose (MC). In the adhesion test, the particles were placed on rat jejunum or stomach in a humid environment in vitro.

The percentage of particles retained on the tissue was considered to be an index of bioadhesion. An in situ perfused ileal loop was used in the rat to study the mucoadhesion of multiple-unit bioadhesive systems. Microspheres of poly (2hydroxyethyl methacrylate) $(315-400 \mu \mathrm{m})$ were synthesized by suspension polymerization and coated with several mucoadhesive polymers in an air-suspension process. The polymer-coated microspheres were perfused with isotonic saline, and the number of collected particles was counted. Based on the mean residence time (MRT) calculated from the residence curves of the particles, the PC coated particles exhibited strong 
mucoadhesive properties. They also proposed an in-vitro model for intestinal absorption testing with rat intestine that was applicable to adhesion studies 54

\subsection{Mucoadhesive Properties of Polymeric NPS} Coated with Mucoadhesive Polymers: The mucoadhesiveness of surface-modified polymeric NPs was also evaluated using the isolated rat intestinal sac ${ }^{55}$. As it is difficult to count the number of the submicron sized particles in suspension, the amount of adhering NPs was measured fluorophotometrically by using fluorescence labelled nanospheres. In this test, the everted rat intestinal sac was immersed in a suspension of fluorescence-labeled nanospheres and gently agitated for $15 \mathrm{~min}$. Then, the amount of NPs adhering to the sac was determined fluorophotometrically after acetone extraction. The CS coated nanospheres showed higher mucoadhesion to the everted intestinal sac in saline than the uncoated nanospheres or nanospheres with other polymer coatings. The mucoadhesive properties of polymer-coated NPs were also evaluated using mucin particles in vitro ${ }^{56}$.

The effectiveness of mucoadhesive polymeric nanospheres was evaluated in the absorption of a peptide drug by using Elcatonin-loaded PLGA nanospheres coated with $\mathrm{CS}{ }^{57}$. The physiological action, i.e. reduction in blood calcium, was monitored after oral administration of the uncoated and CS coated PLGA nanospheres in rat. The blood calcium fell temporarily by about $80-85 \%$ for $1 \mathrm{~h}$ after intragastrical administration of uncoated nanospheres containing elcatonin. The blood calcium-time profile was almost the same as that following the administration of elcatonin solutions of $250 \mathrm{IU} / \mathrm{Kg}$ or less. On increasing the dose of the uncoated nanospheres, the reduction in $\mathrm{Ca}$ concentration was prolonged over $10 \mathrm{~h}$. On the other hand, a significantly prolonged reduction in blood $\mathrm{Ca}$ was obtained over $12 \mathrm{~h}$ after administration of the CS coated nanospheres at doses of $125 \mathrm{IU} / \mathrm{Kg}$ and $250 \mathrm{IU} / \mathrm{Kg}$. On increasing the dose, e.g. to $500 \mathrm{IU} / \mathrm{Kg}$, the reduction in calcium was prolonged.

4.5. Clinical Practice of NPs: The application of nanotechnology to drug delivery has already had a significant impact on many areas of medicine. In recent years, the U.S. Food and Drug Administration (USFDA) has approved Investigational New Drug (IND) applications for nano-formulations, enabling clinical trials for breast, gynecological, solid tumour, lung, mesenchymal tissue, lymphoma, central nervous system and genito-urinary cancer treatments ${ }^{58}$.

A global survey conducted by the European Science and Technology Observatory in 2006 showed that more than 150 companies are developing nanoscale therapeutics. So far, 24 nanotechnology based therapeutic products have been approved for clinical use, with total sales exceeding $\$ 5.4$ billion. Among these products, liposomal drugs and polymer-drug conjugates are two dominant classes, accounting for more than $80 \%$ of the total amount ${ }^{59}$. The recent allocation of $\$ 144$ million by the National Cancer Institute and \$54 million by the National Heart, Lung and Blood Institute in U.S.A. for nanotechnology research in 2004 and 2005 respectively, together with a sharp increase over the past 5 years in the number of patent applications in this area.

Several nanoscale imaging platforms have also been introduced and are currently under pre-clinical or clinical investigation. The most characterized system is iron oxide nanoparticles for use in conjugation with Magnetic Resonance Imaging (MRI) for enhanced resolution imaging. Combidex which is in late stage clinical evaluation is a formulation of iron oxide nanoparticles which has been shown to be significantly more sensitive for detection of prostate cancer lymph node metastasis as compared to conventional MRI ${ }^{60,61}$.

CONCLUSION: Mucoadhesive drug delivery systems (MDDS) are desirable since they provide opportunity for improving drug bioavailability, via an intimate and extended contact between drug and absorbing membrane, and also provide patient compliance, via reduction in dosage frequency and drug induced side effects. Transit of the MDDS through the GIT is mainly governed by mucus turnover, which may increase upon interaction with bioadhesives. Nanosuspension technology and the mucoadhesive principle were combined to develop a formulation for the poorly soluble drug. Mucoadhesive nanoparticulate systems, such as CS-and Carbopol-coated liposomes and PLGA 
NPs, were found to be useful peptide (insulin, calcitonin) carriers for improving oral mucosal delivery due to their prolonged retention in the gastro-intestinal tract and excellent penetration into the mucus layer.

Furthermore, mucoadhesive PLGA NPs were shown to be useful for the pulmonary delivery of peptide drugs as well as oral administration due to sharing the same mechanism as in the enteral absorption of peptides. By incorporation of the nanosuspension into mucoadhesive hydrogels, the physical stability of this system could be increased compared with the caking nanosuspensions, leading to long-term stable systems.

ACKNOWLEDGEMENT: The authors would like to thank the Director, Gujarat Forensic Science University, Institute of Research and Development, and Deputy Drug Controller (India) for their valuable support.

\section{CONFLICT OF INTEREST: Declared none.}

\section{REFERENCES:}

1. Fang L, Wang L, Yao Y, Zhang J, Wu X, Li X, Wang H, Zhang $\mathrm{X}$, Gong $\mathrm{X}$ and Chang J: Micro- and nano-carrier systems: The non-invasive and painless local administration strategies for disease therapy in mucosal tissues. Nanomed: Nanotech Biol Med 2017; 13(1): 153-171.

2. Duggan S, Cummins W, O'Donovan O, Hughes $\mathrm{H}$ and Owens E: Thiolated polymers as mucoadhesive drug delivery systems. Eur J Pharm Sci 2017; 100(30): 64-78.

3. Liu M, Zhang J, Shan W and Huang Y: Developments of mucus penetrating nanoparticles. Asian J Pharm Sci 2015; 54: 212-231.

4. Gu M, Yildiz H, Carrier R and Belfort G: Discovery of low mucus adhesion surfaces. Acta Biomater 2013; 9: 5201-5207.

5. Wang Y, Li P and Kong L: Chitosan-modified PLGA nanoparticles with versatile surface for improved drug delivery. AAPS Pharm Sci Tech 2013; 14: 585-592.

6. Rana P and Murthy RS: Formulation and evaluation of mucoadhesive buccal films impregnated with carvedilol nanosuspension: a potential approach for delivery of drugs having high first-pass metabolism. Drug Deliv 2013; 11: 351-360.

7. Patel DJ and Patel JK: Design and evaluation of famotidine mucoadhesive nanoparticles for aspirin induced ulcer treatment. Braz Arch Biol Technol 2013; 56: 223-236.

8. Park CG, Kim MJ, Park M, Choi SY, Lee SH, Lee JE, Shin GS, Park KH and Choy YB: Nanostructured mucoadhesive microparticles for enhanced preocular retention. Acta Biomater 2014; 10(1): 77-86.

9. Elgadir MA, Uddin MS, Ferdosh S, Adam A, Chowdhury AJK and Sarker MZI: Impact of chitosan composites and chitosan nanoparticle composites on various drug delivery systems: A review. J Food Drug Anal 2015; 23(4): 619629.
10. Liang J, Yan H, Puligundla P, Gaoa X, Zhoua $Y$ and Wan $\mathrm{X}$ : Applications of chitosan nanoparticles to enhance absorption and bioavailability of tea polyphenols: A review. Food Hydrocolloids 2017; 69: 286-292.

11. Ribeiro AJ, Lucena de Souza FR, Bezerra JMNA, Oliveira C, Nadvorny D, de La Roca Soares MF, Nunes LCC, Silva-Filho EC and Veiga F: Gums' based delivery systems: Review on cashew gum and its derivatives. Carbohydrate Polymers 2016; 147: 188-200.

12. Das B, Dutta S, Nayak AK and Nanda U: Zinc alginatecarboxymethyl cashew gum microbeads for prolonged drug release: development and optimization. Int J Biol Macromol 2014; 70: 506-515.

13. Li L, Ni R, Shao Y and Mao S: Carrageenan and its applications in drug delivery. Carbohydr Polym 2014; 103: $1-11$.

14. Prajapati VD, Jani GK, Moradiya NG and Randeria NP: Pharmaceutical applications of various natural gums: mucilages and their modified forms. Carbohydr Polym 2013; 92: 1685-1699.

15. Yang J, Han S, Zheng H, Dong H and Liu J: Preparation and application of micro/nanoparticles based on natural polysaccharides. Carbohydr Polym 2015; 123: 53-66.

16. Raj BS, Shanthi, Nair RS, Samraj PI and Vidya: Formulation and evaluation of coated microspheres for colon targeting. J Appl Pharm Sci 2013; 3(8): S68-S74.

17. Mansuri S, Kesharwani P, Jain K, Tekade RK and Jain NK: Mucoadhesion: A promising approach in drug delivery system. React Func Polym 2016; 100: 151-172.

18. Miladi K, Sfar S, Fessi H and Elaissari A: Enhancement of alendronate encapsulation in chitosan nanoparticles. J Drug Deliv Sci Technol 2015; 30: 391-396.

19. Lü JM, Wang X, Marin-Muller C, Wang H, Lin PH, Yao $\mathrm{Q}$ and Chen $\mathrm{C}$ : Current advances in research and clinical applications of PLGA-based nanotechnology. Expert Rev Mol Diagn 2014; 9: 325-341.

20. Netsomboon K and Bernkop-Schnürch A: Mucoadhesive vs. mucopenetrating particulate drug delivery. Eur J Pharm Biopharm 2016; 98: 76-89.

21. Bugnicourt $\mathrm{L}$ and Ladavière $\mathrm{C}$ : Interests of chitosan nanoparticles ionically cross-linked with tripolyphosphate for biomedical applications. Prog Polym Sci 2016; 60: 1-17.

22. Sosnik A, das Neves J and Sarmento B: Mucoadhesive polymers in the design of nano-drug delivery systems for administration by non-parenteral routes: A review. Prog Polym Sci 2014; 39(12): 2030-2075.

23. Zhang X, Yang X, Ji J, Liu A and Zhai G: Tumor targeting strategies for chitosan-based nanoparticles. Colloids Surf B: Biointerfaces 2016; 148: 460-473.

24. Ensign LM, Cone R and Hanes J: Nanoparticle-based drug delivery to the vagina: a review. J Control Release 2014; 190: 500-514.

25. Caramella CM, Rossi S, Ferrari F, Bonferoni MC and Sandri G: Mucoadhesive and thermogelling systems for vaginal drug delivery. Adv Drug Del Rev 2015; 92(15): 39-52.

26. Chappell CA, Rohan LC, Moncla BJ, Wang L, Meyn LA, Bunge $\mathrm{K}$ and Hillier SL: The effect of reproductive hormones on the physical properties of cervicovaginal fluid. Am J Obstet Gynecol 2014; 211: 226.

27. Senyiğit ZA, Karavana SY, Eraç B, Gürsel O, Limoncu $\mathrm{MH}$ and Baloğlu E: Evaluation of chitosan based vaginal bioadhesive gel formulations for antifungal drugs. Acta Pharma 2014; 64: 139-156.

28. Abruzzo A, Bigucci F, Cerchiara T, Saladini B, Gallucci MC, Cruciani F, Vitali B and Luppi B: Chitosan/alginate complexes for vaginal delivery of chlorhexidine digluconate. Carbohydr Polym 2013; 91: 651-658. 
29. Szymanska E, Winnicka K, Amelian A and Cwalina U: Vaginal chitosan tablets with clotrimazole-design and evaluation of mucoadhesive properties using porcine vaginal mucosa, mucin and gelatin. Chem Pharm Bull 2014; 62: 160-167.

30. Rastogi R, Teller RS, Mesquita PMM, Herold BC and Kiser PF: Osmotic pump tablets for delivery of antiretrovirals to the vaginal mucosa. Antivir Res 2013; 100: 255-258.

31. Bergin K, Suljaković S, Škalko-Basnet N and Kristl A: Mucoadhesive liposomes as new formulation for vaginal delivery of curcumin. Eur J Pharm Biopharm 2014; 87: 40-46.

32. El-Say KM and El-Sawy HS: Polymeric nanoparticles: Promising platform for drug delivery. Int J Pharm 2017; 528(1-2): 675-691.

33. Wattendorf $U$ and Merkle HP: PEGylation as a tool for the biomedical engineering of surface modified microparticles. J Pharm Sci 2008; 97(11): 4655-69.

34. Garcia-Fuentes $M$ and Alonso MJ: Chitosan-based drug nanocarriers: where do we stand? J Control Release 2012; 161: 496-504.

35. Grenha A: Chitosan nanoparticles: a survey of preparation methods. J Drug Target 2012; 20: 291-300.

36. Bugnicourt L, Alcouffe $\mathrm{P}$ and Ladavière $\mathrm{C}$ : Elaboration of chitosan nanoparticles: favorable impact of a mild thermal treatment to obtain finely divided, spherical, and colloiddally stable objects. Colloids Surf A 2014; 457: 476-486.

37. Rampino $\mathrm{A}$, Borgogna $\mathrm{M}$, Blasi $\mathrm{P}$, Bellich $\mathrm{B}$ and Cesàro A: Chitosan nanoparticles: preparation, size evolution and stability. Int J Pharm 2013; 455: 219-228.

38. Soliman GM, Zhang YL, Merle G, Cerruti M and Barralet J: Hydrocaffeic acid-chitosan nanoparticles with enhanced stability, mucoadhesion and permeation properties. Eur $\mathbf{J}$ Pharm Biopharm 2014; 88: 1026-1037.

39. Pahuja P, Arora S and Pawar P: Ocular drug delivery system: a reference to natural polymers. Expert Opin Drug Deliv 2012; 9: 837-861.

40. Chen MC, Mi FL, Liao ZX, Hsiao CW, Sonaje K, Chung MF, Hsu LW and Sung HW: Recent advances in chitosanbased nanoparticles for oral delivery of macromolecules. Adv Drug Deliv Rev 2013; 65: 865-879.

41. $\mathrm{Cu}$ Y, Booth CJ and Saltzman WM: In vivo distribution of surface modified PLGA nanoparticles following intravaginal delivery. J Controlled Release 2011; 156: 258-264.

42. Gan L, Wang J, Jiang M, Bartlett H, Ouyang D, Eperjesi F, Liu J and Gan Y: Recent advances in topical ophthalmic drug delivery with lipid-based nanocarriers. Drug Discov Today 2013; 18: 290-297.

43. Soliman GM, Zhang YL, Merle G, Cerruti M and Barralet J: Hydrocaffeic acid-chitosan nanoparticles with enhanced stability, mucoadhesion and permeation properties. Eur $\mathrm{J}$ Pharm Biopharm 2014; 88: 1026-1037.

44. Saharan V, Mehrotra A, Khatik R, Rawal P, Sharma SS and Pal A: Synthesis of chitosan based nanoparticles and their in vitro evaluation against phytopathogenic fungi. Int J Biol Macromol 2013; 62: 677-683.

45. Dong Y, Ng WK, Shen S, Kim S and Tan RBH: Scalable ionic gelation synthesis of chitosan nanoparticles for drug delivery in static mixers. Carbohydr Polym 2013; 94: 940945.
46. Zhang H, Jung J and Zhao Y: Preparation, characterization and evaluation of antibacterial activity of catechins and catechins-Zn complex loaded B-chitosan nanoparticles of different particle sizes. Carbohydr Polym 2016; 137: 8291.

47. Jino JA and Smith A: Preparation and evaluation of stavudine loaded chitosan nanoparticles. J Pharm Res 2013; 6: 268-274.

48. Joseph JJ, Sangeetha D and Gomathi T: Sunitinib loaded chitosan nanoparticles formulation and its evaluation. Int $\mathbf{J}$ Biol Macromol 2016; 82: 952-958.

49. Ensign LM, Cone R and Hanes J: Oral drug delivery with polymeric nanoparticles: the gastrointestinal mucus barriers. Adv Drug Deliv Rev 2012; 64: 557-570.

50. Lee KY and Mooney DJ: Alginate: properties and biomedical applications. Prog Polym Sci 2012; 37: 106126.

51. Najafabadi AH, Abdouss M and Faghihi S: Synthesis and evaluation of PEG-O-chitosan nanoparticles for delivery of poor water soluble drugs: ibuprofen. Mater Sci Eng C 2014; 41: 91-99.

52. Neelagiri R, Reddy MS, Rao NGR: Buccal patch as drug delivery system: an overview. Int J Curr Pharm Res 2013; 5(2): 40-47.

53. Palacio ML and Bhushan B: Bioadhesion: a review of concepts and applications. Philos Trans A Math Phys Eng Sci 2012; 370: 2321-2347.

54. Ensign LM, Cone R and Hanes J: Oral drug delivery with polymeric nanoparticles: the gastrointestinal mucus barriers. Adv Drug Deliv Rev 2012; 64: 557-570.

55. Patil SB, Kaul A, Babbar A, Mathur R, Mishra A and Sawant KK: In vivo evaluation of alginate microspheres of carvedilol for nasal delivery. J Biomed Mater Res B Appl Biomater 2012; 100: 249-255.

56. Lim JH, You SK, Baek JS, Hwang CJ, Na YG, Shin SC and Cho CW: Surface-modified gemcitabine with mucoadhesive polymer for oral delivery. J Microencapsul 2012; 29: 487-496.

57. Vasi AM, Popa MI, Tanase EC, Butnaru M and Verestiuc L: Poly (acrylic acid)-poly(ethylene glycol) nanoparticles designed for ophthalmic drug delivery. J Pharm Sci 2014; 103: 676-686.

58. Aburahma MH and Mahmoud AA: Biodegradable ocular inserts for sustained delivery of brimonidine tartarate: preparation and in vitro / in vivo evaluation. AAPS PharmSciTech 2011; 12: 1335-1347.

59. Kanazawa T, Taki H, Tanaka K, Takashima Y and Okada $\mathrm{H}$ : Cell-penetrating peptide-modified block copolymer micelles promote direct brain delivery via intranasal administration. Pharm Res 2011; 28: 2130-2139.

60. Sanjai C, Kothan S, Gonil P, Saesoo S and Sajomsang W: Chitosan-triphosphate nanoparticles for encapsulation of super-paramagnetic iron oxide as an MRI contrast agent. Carbohydr Polym 2014; 104: 231-237.

61. Bhosale RR, Gangadharappa HV, Hani U, Osmani RA, Vaghela R, Kulkarni PK and Venkata KS: Current perspectives on novel drug delivery systems and therapies for management of prostate cancer: an inclusive review. Curr Drug Targets 2016.

How to cite this article:

Shankar G and Agrawal YK: A review on nanoparticulate mucoadhesive system. Int J Pharm Sci Res 2017; 8(12): 4983-91.doi: 10.13040/ IJPSR.0975-8232.8(12).4983-91.

All $\odot 2013$ are reserved by International Journal of Pharmaceutical Sciences and Research. This Journal licensed under a Creative Commons Attribution-NonCommercial-ShareAlike 3.0 Unported License.

This article can be downloaded to ANDROID OS based mobile. Scan QR Code using Code/Bar Scanner from your mobile. (Scanners are available on Google Playstore) 